\title{
Selection of silk-binding peptides by phage display
}

\author{
Yoko Nomura • Vandana Sharma • \\ Asami Yamamura $\cdot$ Yohei Yokobayashi
}

Received: 1 December 2010/Accepted: 5 January 2011/Published online: 23 January 2011

(C) The Author(s) 2011. This article is published with open access at Springerlink.com

\begin{abstract}
Peptides that bind to silkworm-derived silk fibroin fiber were selected from a phagedisplayed random peptide library. The selected silkbinding peptides contained a consensus sequence QSWS which is important for silk-binding as confirmed by binding assays using phage and synthetic peptides. With further optimization, we anticipate that the silk-binding peptides will be useful for functionalization of silk for biomaterial applications.
\end{abstract}

Keywords Fibroin fiber - Peptide - Phage display · Silk

\section{Introduction}

Silkworm silk is composed of two classes of proteins fibroin and sericin. It is one of the oldest and most versatile biomaterials with many favorable properties such as biocompatibility and biodegradability. Silk is also easily processed into various forms such as fibers, yarns, textiles, sponges, and films (Altman et al. 2003). Not only has silk been extensively used as sutures in medical settings for centuries but silk-based

Y. Nomura · V. Sharma · A. Yamamura .

Y. Yokobayashi $(\bowtie)$

Department of Biomedical Engineering, University

of California, Davis, 451 Health Sciences Drive,

Davis, CA 95616, USA

e-mail: yoko@ucdavis.edu biomaterials have been developed for modern biomedical applications such as matrices for tissue engineering (Altman et al. 2003; Wang et al. 2006).

Molecular functionalization of silk is an attractive strategy for further expanding the versatility of silk as biomaterials. However, relatively few previous reports on chemical functionalization of silk-derived materials can be found. Most of these efforts involve covalent chemical modifications of the silk proteins using reactive reagents (Murphy and Kaplan 2009). Genetic modifications of silkworms have also been recently reported (Mori and Tsukada 2000). However, genetically modified organisms are still subject to stringent regulatory approval, and the possible modifications are limited to those that can be genetically encoded and nontoxic to the host silkworm.

New universal molecular tools such as peptide tags that can be fused to other functional moieties (e.g. proteins, organic molecules, or nanoparticles) to impart new functions to silk via noncovalent interactions would be highly attractive. Affinity selection using phage libraries that display random peptides fused to a coat protein has proven to be a highly successful strategy for discovering potent peptide ligands (Pande et al. 2010). Many peptide ligands with affinities to various biomolecules (Pande et al. 2010) and inorganic (Sarikaya et al. 2003) and organic materials (Serizawa et al. 2005) have been reported. Here, we describe our initial results of the selection and screening of peptide ligands that bind to silk fibroin fibers from a phage-displayed random 
peptide library. Sequencing of the selected phage clones revealed a short consensus sequence QSWS which appears to be important for binding based on binding assays using phage and synthetic peptides. We envision that these peptide ligands will be useful for noncovalent functionalization of various silkbased biomaterials.

\section{Materials and methods}

Silk degumming

To obtain silk fibroin fiber, Bombyx mori cocoons were degummed by the standard soap/soda ash method (Robson 1999). Briefly, B. mori silk cocoons were gently agitated in degumming solution containing $0.08 \%(\mathrm{w} / \mathrm{v}) \mathrm{Na}_{2} \mathrm{CO}_{3}$ and $0.2 \%$ (w/v) Marseille soap (olive oil soap) at $98^{\circ} \mathrm{C}$ for $1 \mathrm{~h}$. The fibers were rinsed with warm distilled water for $10 \mathrm{~min}$. The degumming process was repeated under a milder condition $\left(0.06 \% \quad \mathrm{Na}_{2} \mathrm{CO}_{3}\right.$ and $0.15 \%$ Marseille soap). The degummed fibers were repeatedly rinsed with warm water for $10 \mathrm{~min}$ and finally rinsed with cold water. The degummed fibers were put on a layer of clean KimWipes to remove excess water and dried at room temperature. The degumming process resulted in $25-30 \%$ loss of the silk weight.

Selection and identification of silk-binding phage

A commercially available M13 phage library (Ph.D.12. New England Biolabs) that displays random 12-mer peptides fused to the minor coat protein pIII was used for peptide selection. Dry degummed silk fibroin fibers $(10 \mathrm{mg})$ were placed in a spin-filter column (USA Scientific) and washed with $500 \mu \mathrm{l}$ of $0.1 \%$ TBST buffer $(50 \mathrm{mM}$ Tris/ $\mathrm{HCl} \mathrm{pH} 7.5$,
$150 \mathrm{mM} \mathrm{NaCl}, 0.1 \%$ v/v Tween 20) using a microcentrifuge $(\sim 1,000 \times g, 10 \mathrm{~s})$. The fibers were incubated with phage in $1 \mathrm{ml} 0.1 \%$ TBST at room temperature with gentle shaking (Table 1). Subsequently, the fibers were transferred to a spin-column and the unbound phage were removed by centrifugation $(\sim 1,000 \times g, 10 \mathrm{~s})$ and washed with $1 \mathrm{ml} 0.1 \%$ TBST. Bound phage were released from the fibers by incubation in $1 \mathrm{ml} 0.2 \mathrm{M}$ glycine/ $\mathrm{HCl}$ buffer $(\mathrm{pH} 2.2$ ) containing $1 \mathrm{mg}$ bovine serum albumin for $5 \mathrm{~min}$ in a fresh tube and separated from the fibers by centrifugation. The solution containing the eluted phage was neutralized by adding $150 \mu \mathrm{l} 1 \mathrm{M}$ Tris/ $\mathrm{HCl}(\mathrm{pH} 9.1)$. The recovered phage were titered or amplified according to the manufacturer's protocol (New England Biolabs) for subsequent rounds of selection. Selection conditions for the subsequent rounds are summarized in Table 1. Phage DNAs were isolated after the third (12 clones) and the fifth (11 clones) rounds of selection and sequenced using $-96 \mathrm{gIII}$ primer (5'-CCCTCATAGTTAGCGTAACG-3').

\section{Phage binding assay}

A phage clone, YN42 identified after the fifth round selection, and its mutants were examined for their silk-binding properties. Phage $\left(5 \times 10^{7} \mathrm{pfu}\right)$ were diluted in $0.1 \%$ TBST $(1 \mathrm{ml})$ and incubated with silk fibroin fibers $(10 \mathrm{mg}$ ) for $15 \mathrm{~min}$ at room temperature with gentle shaking. The fibers were washed 10 times with $0.5 \%$ TBST $(1 \mathrm{ml})$. Bound phage were eluted as described above and titered according to the manufacturer's protocols.

Synthetic peptide binding assay

Peptides were chemically synthesized with $C$-terminal biotin by LifeTein. Each 12-mer peptide was

Table 1 Summary of phage selection conditions

\begin{tabular}{llllr}
\hline Selection round & Input phage $^{\mathrm{a}}(\mathrm{pfu})$ & Recovered phage $^{\mathrm{b}}(\mathrm{pfu})$ & Binding time $(\mathrm{min})^{\text {Number of washes }}$ \\
\hline 1 & $4 \times 10^{10}$ & $4.3 \times 10^{7}$ & 90 & 2 \\
2 & $2 \times 10^{11}$ & $9.1 \times 10^{7}$ & 90 & 5 \\
3 & $2 \times 10^{11}$ & $4.3 \times 10^{7}$ & 30 & 10 \\
4 & $2 \times 10^{11}$ & $4.4 \times 10^{6}$ & 15 & 10 \\
5 & $2 \times 10^{11}$ & $3.7 \times 10^{7}$ & 15 & 10 \\
\hline
\end{tabular}

a, b Phage counts are given in plaque-forming units (pfu). For the first round, 0.1\% TBST was used as the washing buffer. For the second through fifth round, $0.5 \%$ TBST was used 
synthesized with GK at its $C$-terminus with the terminal lysine side chain biotinylated. The peptides were synthesized with $N$-terminal amine and $C$-terminal amides, and HPLC purified to $>86 \%$ purity. Peptide solution at an appropriate concentration $(400 \mu \mathrm{l})$ in $0.1 \%$ TBST was incubated with silk fibers $(1 \mathrm{mg})$ for $30 \mathrm{~min}$ at room temperature with gentle shaking. After washing the fibers ten times with $400 \mu \mathrm{l}$ of $0.5 \%$ TBST, the fibers were blocked with $1 \%(\mathrm{w} / \mathrm{v})$ BSA in TBS $(400 \mu \mathrm{l})$ for $2 \mathrm{~h}$. After washing the fibers five times with TBS $(400 \mu \mathrm{l})$, the fibers were incubated with $400 \mu \mathrm{l}$ streptavidin-horse radish peroxidase (HRP) conjugate (Rockland) solution (diluted 1:20,000 in TBS) for $1 \mathrm{~h}$. The fibers were further washed six times with $0.1 \%$ TBST and transferred to a fresh tube. Subsequently, HRP substrate solution $(500 \mu \mathrm{l})$ containing ABTS $(0.4 \mathrm{mg} / \mathrm{ml})$ and $\mathrm{H}_{2} \mathrm{O}_{2}(0.03 \%$ w/v) was added to the fibers and reacted for $30 \mathrm{~min}$ at room temperature with gentle shaking. The fibers were removed and $\mathrm{A}_{405}$ and $\mathrm{A}_{495}$ were measured using a microplate reader. The ELISA signals were calculated as $\mathrm{A}_{405}-\mathrm{A}_{495}$ normalized by the weight of the fibers. Background signal obtained without peptide was subtracted.

\section{Results and discussion}

Selection and identification of silk-binding peptides

Results of the phage selection process are summarized in Table 1. Stringency of selection was increased each round by decreasing the binding time and/or increasing the number of washes, except for the fifth round in which the binding and washing conditions were kept the same as the fourth round. We noticed that the number of phage recovered decreased by an order of magnitude after the fourth round, probably due to the higher stringency. However, the phage recovery increased in the fifth round suggesting that the clones with higher affinities to silk fiber were being selected. We randomly picked and analyzed the sequences of 12 clones from the phage selected after the third round. However, the result revealed no consensus or enriched sequences (data not shown). On the other hand, 11 clones analyzed after the fifth round revealed two sequences YN42 and YN43 that appeared three times
Table 2 Amino acid sequences of the phage-displayed peptides after silk affinity selection

\begin{tabular}{lll}
\hline Phage $^{\text {a }}$ & Number of clones & Sequence \\
\hline YN42 & 3 & SYTFHWHQSWSS \\
YN43 & 3 & QSWSWHWTSHVT \\
YN41 & 1 & WTWRWAHVTNTR \\
YN48 & 1 & QDVHLTQQSRYT \\
YN49 & 1 & HKAHEYDPWISP \\
YN50 & 1 & SYSQHYGIPNPW \\
YN52 & 1 & SSWQMSWSWMGS
\end{tabular}

${ }^{a}$ The sequenced phage clones were randomly picked from the fifth round selection. A total of 11 clones were sequenced

each (Table 2). Moreover, both of these enriched sequences contained a common tetrapeptide sequence QSWS. A closer examination of the selected sequences indicates other characteristic patterns such as the high frequency of tryptophan residues.

\section{Characterization of silk-binding peptides}

We focused our initial studies on one of the two highly enriched peptides YN42, because both peptides (YN42 and YN43) contained the common QSWS motif. First, the silk-binding property of the phage clone displaying the YN42 peptide was confirmed by phage binding assay. YN42 phage were recovered in 100 times better yield than the unselected phage population, indicating that the selection indeed enriched phage displaying silk-binding peptides (Fig. 1). To gauge the contributions, if any, of the QSWS motif, two single mutants of YN42 were constructed that display QAWS or QSAS instead of QSWS. While the recovery of the phage YN42/ QAWS was moderately lower (by approximately $30 \%$ ) compared to YN42, the recovery of YN42/ QSAS phage were reduced by more than $90 \%$, indicating that the tryptophan in QSWS motif plays a significant role in silk-binding.

Results of the peptide binding assays using synthetic biotinylated peptides generally agreed with the phage binding assay (Fig. 2a). In particular, a control peptide with the same amino acid composition but with a randomly shuffled amino acid sequence (pepYN42/Shuffled) showed significantly lower affinity to silk compared to pepYN42, indicating that the sequence, not the amino acid composition, is 


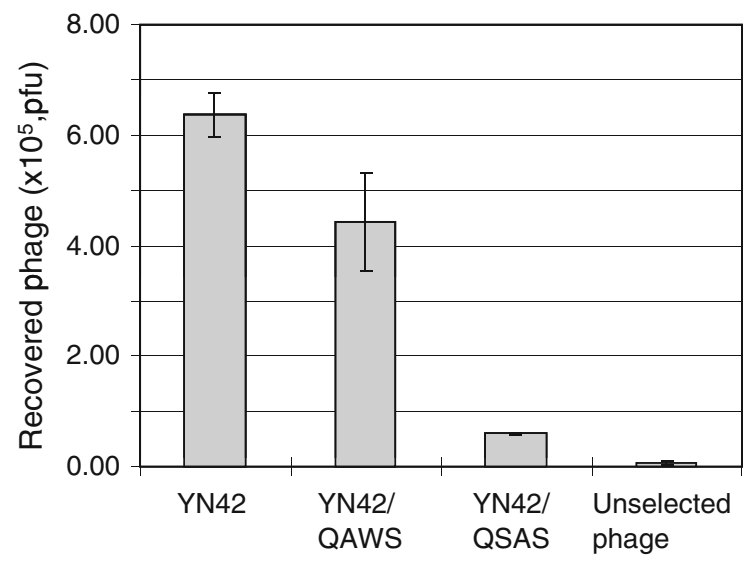

Fig. 1 Phage binding assay. Silk fibroin fibers $(10 \mathrm{mg})$ were incubated with phage $\left(5 \times 10^{7} \mathrm{pfu}\right)$ in $1 \mathrm{ml}$ solution. After washing as described in "Materials and methods" section, bound phage were eluted in $1 \mathrm{ml}$ buffer and titered. The assays were performed twice for each phage clone and the error bars indicate the range of the measurements

important for the observed silk-binding property. To demonstrate how silk-binding peptides can be used to functionalize silk, silk fibers treated with pepYN42 and streptavidin-HRP conjugate were stained by an insoluble substrate $\left(3,3^{\prime}, 5,5^{\prime}\right.$-tetramethylbenzidine, TMB) for HRP. While the fibers treated with pepYN42/Shuffled were only minimally stained, the fibers treated with pepYN42 were stained significantly (Fig. 2b). Concentration dependent binding assay using pepYN42 suggests an apparent submicromolar affinity of the peptide to silk fibroin fibers (Fig. 3).

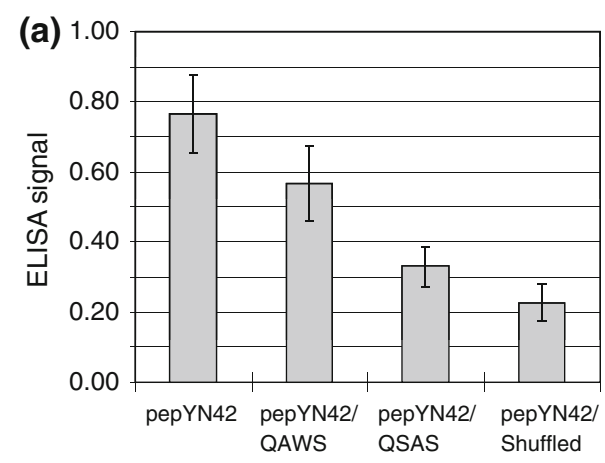

Fig. 2 Synthetic peptide binding assay. Peptides with the amino acid sequence corresponding to the one displayed on the YN42 phage and its variants were synthesized and conjugated to biotin via GK (biotin) at the $C$-terminus. pepYN42/Shuffled: $\mathrm{H}_{2} \mathrm{~N}$ WYSHSHSQTSFWGK(biotin)-CONH 2 . a Peptides $(3 \mu \mathrm{M})$ were incubated with silk fibers $(1 \mathrm{mg})$ and the bound peptides were quantified by ELISA as described in "Materials and

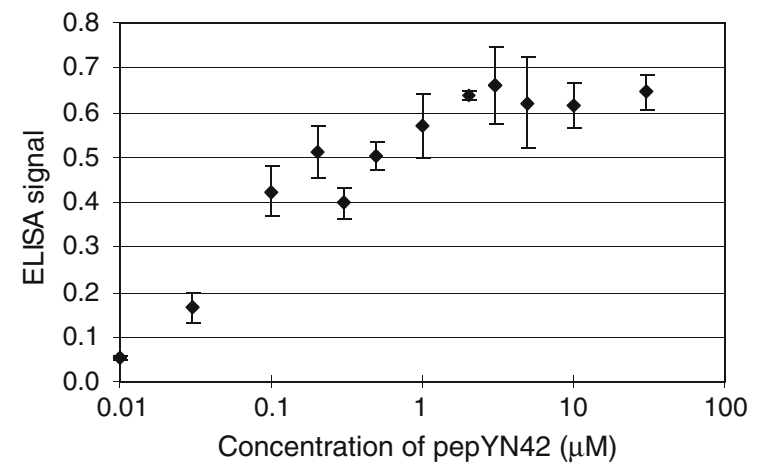

Fig. 3 Concentration dependent binding of pepYN42 to silk fibers. Each data point represents an average of two independent experiments and the error bars indicate the range of the two measurements

\section{Discussion}

Our goal was to discover small peptides with significant affinity to silk fibroin fibers that can be used to noncovalently modify silk-based biomaterials with various functional units such as enzymes, hormones, or signaling molecules. We identified YN42 as one of the two highly enriched peptides in silk affinity selection from a phage displayed peptide library. The YN42 peptide exhibited significant affinity to silk fibroin fiber as a coat-protein fusion displayed on M13 phage, as well as a $C$-terminally biotinylated synthetic peptide. Therefore, YN42 or its derivatives are promising candidates for generic

(b)

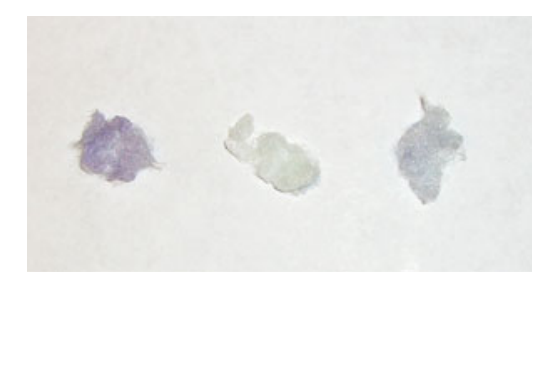

methods" section using ABTS as the chromogenic substrate. The data shown are averages of four replicate experiments and the error bars indicate \pm SD. b Visual detection of biotinylated silkbinding peptides using streptavidin-HRP conjugate and an insoluble HRP substrate (TMB). Left: silk fibers treated with pepYN42; center: untreated fibers; right: fibers treated with pepYN42/Shuffled 
silk-binding tags that can be used to impart new functions to silk-based materials. We anticipate that our silk-binding peptides can be used in a number of forms, for example, as synthetic peptide conjugate demonstrated in this work and as genetically encoded fusion tags to other proteins. Further modifications of the peptide sequence may enable fine-tuning of the silk-binding property for individual applications.

Acknowledgment This research was supported by University of California, Davis through Academic Federation Research Grant Program-Innovative Developmental Award.

Open Access This article is distributed under the terms of the Creative Commons Attribution Noncommercial License which permits any noncommercial use, distribution, and reproduction in any medium, provided the original author(s) and source are credited.

\section{References}

Altman GH, Diaz F, Jakuba C, Calabro T, Horan RL, Chen J, Lu H, Richmond J, Kaplan DL (2003) Silk-based biomaterials. Biomaterials 24:401-416
Mori H, Tsukada M (2000) New silk protein: modification of silk protein by gene engineering for production of biomaterials. J Biotechnol 74:95-103

Murphy AR, Kaplan DL (2009) Biomedical applications of chemically-modified silk fibroin. J Mater Chem 19: 6443-6450

Pande J, Szewczyk MM, Grover AK (2010) Phage display: concept, innovations, applications and future. Biotechnol Adv 28:849-858

Robson RM (1999) Microvoids in Bombyx mori silk. An electron microscope study. Int J Biol Macromol 24: $145-150$

Sarikaya M, Tamerler C, Jen AK, Schulten K, Baneyx F (2003) Molecular biomimetics: nanotechnology through biology. Nat Mater 2:577-585

Serizawa T, Sawada T, Matsuno H, Matsubara T, Sato T (2005) A peptide motif recognizing a polymer stereoregularity. J Am Chem Soc 127:13780-13781

Wang Y, Kim HJ, Vunjak-Novakovic G, Kaplan DL (2006) Stem cell-based tissue engineering with silk biomaterials. Biomaterials 27:6064-6082 